\title{
Aggregation of Spin Labeled Trichogin GA IV Dimers: Distance Distribution Between Spin Labels in Frozen Solutions by PELDOR Data
}

\author{
A. D. Milov, ${ }^{a}$ Yu.D.Tsvetkov, ${ }^{a^{*}}$ F.Formaggio, ${ }^{b}$ S.Oancea,${ }^{b}$ C.Toniolo ${ }^{b}$ and J.Raap ${ }^{c}$
}

${ }^{a}$ Institute of Chemical Kinetics and Combustion, Novosibirsk, 630090 Russian Federation

${ }^{b}$ Institute of Biomolecular Chemistry, CNR, Department of Organic Chemistry, University of Padova, 35131 Padova, Italy

${ }^{c}$ Leiden Institute of Chemistry, Gorlaeus Laboratories, Leiden University, 2300 RA Leiden, The Netherlands 
TLC: Merck $60 \mathrm{~F}_{254}$ plates; eluants: $\mathrm{Rf}_{1}=\mathrm{CHCl}_{3} / \mathrm{EtOH}$ 9:1, $\mathrm{Rf}_{2}=n-\mathrm{BuOH} / \mathrm{AcOH} / \mathrm{H}_{2} \mathrm{O} \quad 6: 2: 2, \mathrm{Rf}_{3}$ $=$ toluene/EtOH 7:1.

\section{Ac-[TOAC ${ }^{1}$, LeuOMe $\left.^{11}\right]$-trichogin}

$\mathrm{H}-\left[\mathrm{TOAC}^{1}\right.$, LeuOMe $\left.^{11}\right]$-trichogin $(0.4 \mathrm{~g}, 0.37 \mathrm{mmol})$, obtained by treating $0.5 \mathrm{~g}(0.38 \mathrm{mmol})$ of Fmoc-[TOAC ${ }^{1}$, LeuOMe ${ }^{11}$-trichogin [1] with an acetonitrile:diethylamine 70:30 mixture, was dissolved in $5 \mathrm{ml}$ of $\mathrm{CH}_{2} \mathrm{Cl}_{2}$ and added with an excess of acetic anhydride. After stirring the reaction mixture overnight, the solvent was removed under reduced pressure. The product crystallized from EtOAc/ PE. Yield 89\%; m.p. $=161-163{ }^{\circ} \mathrm{C} ; \mathrm{Rf}_{1}=0.25, \mathrm{Rf}_{2}=0.95, \mathrm{Rf}_{3}=0.15$; $[\alpha]_{\mathrm{D}^{20}}=-8.4^{\circ}(\mathrm{c}=0.5, \mathrm{MeOH}) ; \operatorname{IR}(\mathrm{KBr}) 3315,1744,1659,1541 \mathrm{~cm}^{-1}$.

\section{Ac-[LeuOMe $\left.{ }^{11}\right]$-trichogin [2]}

$\mathrm{H}$-[LeuOMe ${ }^{11}$-trichogin $(0.5 \mathrm{~g}, 0.52 \mathrm{mmol})$, obtained by Pd-catalyzed hydrogenolysis of $0.6 \mathrm{~g}$ (0.54 mmole) Z-[LeuOMe ${ }^{11}$-trichogin [2] in $\mathrm{MeOH}$, was dissolved in $6 \mathrm{ml}$ of $\mathrm{CH}_{2} \mathrm{Cl}_{2}$. To this solution an excess of acetic anhydride was added. After stirring the reaction mixture overnight, the solvent was removed under reduced pressure. The product crystallized from EtOAc/PE. Yield 89\%; m.p. $=155-158{ }^{\circ} \mathrm{C} ; \mathrm{Rf}_{1}=0.20, \mathrm{Rf}_{2}=0.90, \mathrm{Rf}_{3}=0.10 ;[\alpha]_{\mathrm{D}}{ }^{20}=-20.3^{\circ}(\mathrm{c}=0.1, \mathrm{MeOH}) ; \operatorname{IR}(\mathrm{KBr})$ $3314,1745,1659,1540 \mathrm{~cm}^{-1}$.

\section{Ac- $\left[\right.$ LeuOH $\left.^{11}\right]$-trichogin}

To a solution of Ac-[LeuOMe $\left.{ }^{11}\right]$-trichogin $(0.17 \mathrm{~g}, 0.168 \mathrm{mmol})$ in $\mathrm{MeOH}$ a $2 \mathrm{~N} \mathrm{NaOH}$ solution $(0.36 \mathrm{ml}, 0.72 \mathrm{mmol})$ was added. After stirring at room temperature for $3 \mathrm{~h}$ a $0.1 \mathrm{~N}$ solution of $\mathrm{HCl}$ $(7.2 \mathrm{ml})$ was added and the aqueous phase was extracted several times with $\mathrm{CH}_{2} \mathrm{Cl}_{2}$. The organic phase was washed with water, dried over anhydrous $\mathrm{Na}_{2} \mathrm{SO}_{4}$, and evaporated to dryness. The product crystallized from $\mathrm{CH}_{2} \mathrm{Cl}_{2} / \mathrm{PE}$. Yield 40\%; m.p. $=163-165^{\circ} \mathrm{C} ; \mathrm{Rf}_{2}=0.60 ;[\alpha]_{\mathrm{D}}{ }^{20}=-21.8^{\circ}$ (c $=0.1, \mathrm{MeOH})$; IR (KBr) $3317,1727,1658,1541 \mathrm{~cm}^{-1}$.

\section{Ac-[TOAC ${ }^{1}$, LeuOH $\left.{ }^{11}\right]$-trichogin}

To a solution of Ac-[TOAC ${ }^{1}$, LeuOMe $\left.{ }^{11}\right]$-trichogin $(0.15 \mathrm{~g}, 0.134$ mmole $)$ in $\mathrm{MeOH}$ a $2 \mathrm{~N} \mathrm{NaOH}$ solution $(0.22 \mathrm{ml}, 0.44 \mathrm{mmol})$ was added and the resulting mixture was stirred at room temperature for $7 \mathrm{~h}$. Then, a $0.1 \mathrm{~N} \mathrm{HCl}$ solution $(4.4 \mathrm{ml}, 0.44 \mathrm{mmol})$ was added and the aqueous phase was extracted several times with $\mathrm{CH}_{2} \mathrm{Cl}_{2}$. The organic phase was dried over anhydrous $\mathrm{Na}_{2} \mathrm{SO}_{4}$ and the solvent was removed under reduced pressure. The product crystallized from $\mathrm{CH}_{2} \mathrm{Cl}_{2} / \mathrm{PE}$. Yield $76 \%$; m.p. $=171-172{ }^{\circ} \mathrm{C} ; \mathrm{Rf}_{1}=0.10, \mathrm{Rf}_{2}=0.65 ;[\alpha]_{\mathrm{D}}{ }^{20}=-4.8^{\circ}(\mathrm{c}=0.5, \mathrm{MeOH}) ; \mathrm{IR}(\mathrm{KBr}) 3315$, $1660,1541 \mathrm{~cm}^{-1}$.

\section{Ac-Aib-Gly-L-Leu-Aib-Gly-Gly-L-Leu-Aib-Gly-L-Ile-L-Leu-Aib-Gly-L-Leu-Aib-Gly-Gly-L- Leu-Aib-Gly-L-Ile-L-LeuOMe (DT0)}

To a solution of Ac-[LeuOH $\left.{ }^{11}\right]$-trichogin $(118 \mathrm{mg}, 0.118 \mathrm{mmol})$ in anhydrous $\mathrm{CH}_{2} \mathrm{Cl}_{2}$ at $0{ }^{0} \mathrm{C}$ HOAt (32 mg, $0.142 \mathrm{mmol})$ and $\mathrm{EDC} \cdot \mathrm{HCl}(34 \mathrm{mg}, 0.177 \mathrm{mmol})$ were added. After stirring for 10 min at $0{ }^{\circ} \mathrm{C}$ a solution of $\mathrm{H}$-[LeuOMe ${ }^{11}$-trichogin $(136 \mathrm{mg}, 0.142 \mathrm{mmol})$, obtained by Pd-catalyzed hydrogenolysis in $\mathrm{MeOH}$ of the corresponding Z-[LeuOMe ${ }^{11}$ ]-trichogin (156 mg, $0.142 \mathrm{mmol}$ ) [2], in $\mathrm{CH}_{2} \mathrm{Cl}_{2}$ was added followed by NMM $(20 \mu \mathrm{l}, 0.177 \mathrm{mmol})$. After stirring the reaction at room temperature for 6 days the solvent was removed under reduced pressure. The residue was purified 
by means of RP-HPLC (Vydac $\mathrm{C} 4$ column, $1 \mathrm{~cm}$ diameter, gradient $70-90 \% \mathrm{~B}$ in $30 \mathrm{~min}$., $\mathrm{A}=\mathrm{H}_{2} \mathrm{O}$, $\mathrm{B}=\mathrm{CH}_{3} \mathrm{CN} / \mathrm{H}_{2} \mathrm{O}=9 / 1$, flow $3 \mathrm{ml} / \mathrm{min}$.). Yield 52\%. HPLC purity $100 \%$, $\mathrm{t}_{\mathrm{R}}=15.3 ; \mathrm{m} . \mathrm{p} .=246-247$ ${ }^{\circ} \mathrm{C} ; \mathrm{Rf}_{2}=0.75 ;[\alpha]_{\mathrm{D}}{ }^{20}=-3.2^{\circ}(\mathrm{c}=0.5, \mathrm{MeOH}) ; \mathrm{IR}(\mathrm{KBr}) 3317,1742,1661,1541 \mathrm{~cm}^{-1}$.

\section{Ac-TOAC-Gly-L-Leu-Aib-Gly-Gly-L-Leu-Aib-Gly-L-Ile-L-Leu-Aib-Gly-L-Leu-Aib-Gly-Gly- L-Leu-Aib-Gly-L-Ile-L-LeuOMe (DT1)}

Ac-[TOAC ${ }^{1}, \mathrm{LeuOH}^{11}$ ]-trichogin $(70 \mathrm{mg}, 0.631 \mathrm{mmol})$ was dissolved in anhydrous $\mathrm{CH}_{2} \mathrm{Cl}_{2}$ at $0{ }^{0} \mathrm{C}$. Then, HOAt (12.8 mg, $0.946 \mathrm{mmol})$ and $\mathrm{EDC} \cdot \mathrm{HCl}(18.1 \mathrm{mg}, 0.946 \mathrm{mmol})$ were added, followed, after $10 \mathrm{~min}$, by NMM $(10.5 \mu \mathrm{l}, 0.946 \mathrm{mmol})$ and $\mathrm{CH}_{2} \mathrm{Cl}_{2}$ solution of $\mathrm{H}$-[LeuOMe ${ }^{11}$ ]-trichogin $(92$ $\mathrm{mg}, 0.946 \mathrm{mmol})$, obtained by Pd-catalyzed hydrogenolysis of the corresponding Z-[LeuOMe ${ }^{11}$ ]trichogin (114 mg, $0.103 \mathrm{mmol})$ [2] in $\mathrm{MeOH}$. After stirring the reaction mixture at room temperature for 6 days, the solvent was removed under reduced pressure. The residue was dissolved in EtOAc and washed with $10 \% \mathrm{KHSO}_{4}, \mathrm{H}_{2} \mathrm{O}, 5 \% \mathrm{NaHCO}_{3}, \mathrm{H}_{2} \mathrm{O}$, dried over anhydrous $\mathrm{Na}_{2} \mathrm{SO}_{4}$ and evaporated to dryness under reduced pressure. The residue was first isolated by means of "flash chromatography" by eluting the silica-gel 60 column with a stepwise gradient $\left(\mathrm{CH}_{2} \mathrm{Cl}_{2} / \mathrm{MeOH}\right.$ from 8:1 to 8:2). A further purification step was performed by RP-HPLC (Vydac C4 column, $1 \mathrm{~cm}$ diameter, gradient $70-90 \% \mathrm{~B}$ in 30 min., $\mathrm{A}=\mathrm{H}_{2} \mathrm{O}, \mathrm{B}=\mathrm{CH}_{3} \mathrm{CN} / \mathrm{H}_{2} \mathrm{O}=9 / 1$, flow $2.5 \mathrm{ml} / \mathrm{min}$ ). Yield $37 \%$. HPLC purity $100 \%, \mathrm{t}_{\mathrm{R}}=18.98$; m.p. $=178-180{ }^{\circ} \mathrm{C} ;[\alpha]_{\mathrm{D}}{ }^{20}=+2.0^{\circ}(\mathrm{c}=0.1, \mathrm{MeOH}),[\alpha]_{365}{ }^{20}=$ $-53.3^{\circ}(\mathrm{c}=0.1, \mathrm{MeOH})$; ESI-MS (Finnigan MAT SSQ 710C electrospray spectrometer equipped with an IBM PS/2 95XP 486 in positive ionisation mode): $1030.3\left[\left(\mathrm{M}+2 \mathrm{H}^{+}\right) / 2\right]\left(\mathrm{MW}_{\text {calc: }}: 2058.56\right)$.

\section{Ac-TOAC-Gly-L-Leu-Aib-Gly-Gly-L-Leu-Aib-Gly-L-Ile-L-Leu-Aib-Gly-L-Leu-Aib-Gly-Gly- L-Leu-TOAC-Gly-L-Ile-L-LeuOMe (DT2)}

To a solution of Ac-[TOAC ${ }^{1}$, LeuOH ${ }^{11}$-trichogin $(100 \mathrm{mg}, 0.902 \mathrm{mmol})$ in anhydrous $\mathrm{CH}_{2} \mathrm{Cl}_{2}$ at 0 ${ }^{0} \mathrm{C}$ HOAt $(18.5 \mathrm{mg}, 0.135 \mathrm{mmol})$ and $\mathrm{EDC} \cdot \mathrm{HCl}(25.9 \mathrm{mg}, 0.135 \mathrm{mmol})$ were added, followed, after $10 \mathrm{~min}$, by NMM $(19.8 \mu \mathrm{l}, 0.180 \mathrm{mmol})$ and a $\mathrm{CH}_{2} \mathrm{Cl}_{2}$ solution of $\mathrm{H}$-[TOAC ${ }^{8}$, LeuOMe ${ }^{11}$-trichogin (165 mg, $0.135 \mathrm{mmol}$ ), obtained by treating the corresponding Fmoc-[TOAC ${ }^{8}$, LeuOMe ${ }^{11}$ ]trichogin (200 mg, $0.153 \mathrm{mmol})$ [1] with a $\mathrm{CH}_{2} \mathrm{Cl}_{2} /$ diethylamine $70: 30$ mixture. After stirring the reaction mixture at room temperature for 5 days, the solvent was removed under reduced pressure. The residue was dissolved in EtOAc and washed with $10 \% \mathrm{KHSO}_{4}, \mathrm{H}_{2} \mathrm{O}, 5 \% \mathrm{NaHCO}_{3}, \mathrm{H}_{2} \mathrm{O}$, dried over anhydrous $\mathrm{Na}_{2} \mathrm{SO}_{4}$ and evaporated to dryness under reduced pressure. The residue was first isolated by means of "flash chromatography" by eluting the silica-gel 60 column with a stepwise gradient $\left(\mathrm{CH}_{2} \mathrm{Cl}_{2} / \mathrm{MeOH}\right.$ from 8:1 to 8:2). A further purification step was performed by using preparative RP-HPLC (Vydac C4 column, $1 \mathrm{~cm}$ diameter, gradient 80-100\% B in 30 min., $\mathrm{A}=\mathrm{H}_{2} \mathrm{O}$, $\mathrm{B}=\mathrm{CH}_{3} \mathrm{CN} / \mathrm{H}_{2} \mathrm{O}=9: 1$, flow $2.5 \mathrm{ml} / \mathrm{min}$.). Yield $45 \%$. HPLC purity $100 \%, \mathrm{t}_{\mathrm{R}}=19.65 ; \mathrm{m} . \mathrm{p} .=194-196$ ${ }^{\circ} \mathrm{C} ;[\alpha]_{\mathrm{D}}{ }^{20}=+3.9^{\circ}(\mathrm{c}=0.1, \mathrm{MeOH}),[\alpha]_{365}{ }^{20}=-60.3^{\circ}(\mathrm{c}=0.1, \mathrm{MeOH})$; ESI-MS (Finnigan MAT SSQ 710C electrospray spectrometer equipped with an IBM PS/2 95XP 486 in positive ionisation mode): $1085.8\left[\left(\mathrm{M}+2 \mathrm{H}^{+}\right) / 2\right]\left(\mathrm{MW}_{\text {calc }}: 2170.72\right)$.

[1] Monaco, V.; Formaggio, F.; Crisma, M.; Toniolo, C.; Hanson, P.; Millhauser, G. Biopolymers 1999, 50, 239-253.

[2] Toniolo, C.; Crisma, M.; Formaggio, F.; Peggion, C.; Monaco, V.; Goulard, C.; Rebuffat, S.; Bodo, B. J. Am. Chem. Soc. 1996, 118, 4952-4958. 